\title{
High-resolution gas phase spectroscopy with a distributed feedback terahertz quantum cascade laser
}

Cite as: Appl. Phys. Lett. 89, 061115 (2006); https://doi.org/10.1063/1.2335803

Submitted: 29 March 2006 . Accepted: 23 June 2006 . Published Online: 10 August 2006

H.-W. Hübers, S. G. Pavlov, H. Richter, A. D. Semenov, L. Mahler, A. Tredicucci, H. E. Beere, and D. A. Ritchie

\section{ARTICLES YOU MAY BE INTERESTED IN}

Terahertz gas spectroscopy through self-mixing in a quantum-cascade laser Applied Physics Letters 109, 191101 (2016); https://doi.org/10.1063/1.4967435

Terahertz heterodyne receiver based on a quantum cascade laser and a superconducting bolometer

Applied Physics Letters 86, 244104 (2005); https://doi.org/10.1063/1.1949724

High-resolution heterodyne spectroscopy using a tunable quantum cascade laser around 3.5 $\mathrm{THz}$

Applied Physics Letters 98, 231109 (2011); https://doi.org/10.1063/1.3599518 


\title{
High-resolution gas phase spectroscopy with a distributed feedback terahertz quantum cascade laser
}

\author{
H.-W. Hübers, ${ }^{\text {a) }}$ S. G. Pavlov, H. Richter, and A. D. Semenov \\ German Aerospace Center (DLR), Rutherfordstr. 2, 12489 Berlin, Germany \\ L. Mahler and A. Tredicucci \\ NEST, CNR-INFM and Scuola Normale Superiore, Piazza dei Cavalieri 7, 56126 Pisa, Italy \\ H. E. Beere and D. A. Ritchie \\ Cavendish Laboratory, University of Cambridge, Madingley Road, Cambridge CB3 OHE, United Kingdom
}

(Received 29 March 2006; accepted 23 June 2006; published online 10 August 2006)

\begin{abstract}
The quantum cascade laser is a powerful, narrow linewidth, and continuous wave source of terahertz radiation. The authors have implemented a distributed feedback device in a spectrometer for high-resolution gas phase spectroscopy. Amplitude as well as frequency modulation schemes have been realized. The absolute frequency was determined by mixing the radiation from the quantum cascade laser with that from a gas laser. The pressure broadening and the pressure shift of a rotational transition of methanol at $2.519 \mathrm{THz}$ were measured in order to demonstrate the performance of the spectrometer. (C) 2006 American Institute of Physics. [DOI: 10.1063/1.2335803]
\end{abstract}

High-resolution gas phase spectroscopy at terahertz frequencies is a powerful tool for investigations of the structure and energy levels of molecules and atoms. Besides information on the species itself, important information on Doppler and pressure broadening can be obtained from terahertz spectra. These data are a prerequisite for the interpretation of spectra obtained from astronomical sources or planetary atmospheres including the Earth. ${ }^{1}$ In the low terahertz region different methods have been developed, which are mostly based on fundamental microwave oscillators (for example, backward wave oscillators or klystrons) or harmonic generation from these sources. ${ }^{2}$ In contrast, spectroscopy above $2 \mathrm{THz}$ is hampered by the lack of frequency tunable, continuous wave, powerful, and narrow linewidth radiation sources. Several technologies providing tunable terahertz radiation have been demonstrated. One approach is to combine the radiation from two $\mathrm{CO}_{2}$ lasers in a metal-insulator-metal diode, which generates a difference frequency in the terahertz range. Frequency tunability of about $40 \mathrm{GHz}$ is achieved by adding a tunable microwave source. ${ }^{3}$ The drawbacks of this technique are the small amount of terahertz power (typically a few tenths of a microwatt) and the need for a sophisticated frequency stabilization of the $\mathrm{CO}_{2}$ lasers. Another approach is based on sideband generation by mixing the radiation of an optically pumped terahertz gas laser with radiation of a microwave source or a backward wave oscillator in a Schottky barrier diode. ${ }^{4-6}$ This yields more but still little output power (typically several microwatt) and again suffers from complexity. In addition, the frequency coverage is limited because only powerful gas laser lines can be used for sideband generation. The sensitivity of an instrument relying on either of these techniques is ultimately limited by the available power. Terahertz time domain spectroscopy is an alternative technology which is based on lasers emitting femtosecond pulses. These are used to generate broadband (up to several terahertz) pulses. ${ }^{7,8}$ However, the spectral resolution of this technique is typically $1-10 \mathrm{GHz}$. The most

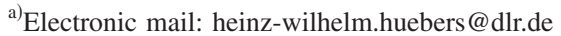

sensitive method is laser magnetic resonance spectroscopy that makes use of the Zeeman effect but is limited to paramagnetic species. ${ }^{9}$ The recently developed terahertz quantum

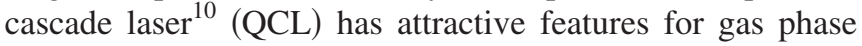
spectroscopy, namely, its intrinsic linewidth of less than $20 \mathrm{kHz}$ (Refs. 11-13) and high output power. ${ }^{14}$ In this letter we describe a high-resolution terahertz spectrometer based on a QCL and present first spectroscopic results.

The QCL is designed for an operation frequency around 2.5 THz. It is based on a GaAs/AlGaAs superlattice and a plasmon-type waveguide. The design follows the so-called bound-to-continuum approach. ${ }^{15}$ Design and fabrication are identical with those described in Ref. 16. The active medium is formed by 110 repeat units of the superlattice (total thickness of $15 \mu \mathrm{m}$ ) covered on top by a $\mathrm{Cr} / \mathrm{Au}$ layer. This layer is patterned into a series of narrow slits with half-wavelength period to create a distributed feedback (DFB) structure. The other boundary of the waveguide consists of two highly doped GaAs layers located between the substrate and the active medium. The resonator is a mesa-etched, $240 \mu \mathrm{m}$ wide ridge with a length of $2.5 \mathrm{~mm}$ defined by cleaving. The laser is soldered to a copper bar, wire bonded, and mounted in a holder with a Winston cone $(f$ number $=6)$ close to the outcoupling facet of the laser. The QCL is operated in a mechanical cryocooler (model SRDK-408D from Sumitomo Heavy Industries Ltd.). In order to minimize vibrations it is mechanically isolated from the coldfinger by copper wires. The cooler has a heat extraction capacity of $1 \mathrm{~W}$ at $4.2 \mathrm{~K}$. Since the input power of the laser is $5-10 \mathrm{~W}$, the smallest achievable temperature at the position of the QCL is $\sim 20 \mathrm{~K}$ during laser operation. The laser threshold is about $80 \mathrm{~A} / \mathrm{cm}^{2}$ at $20 \mathrm{~K}$, the maximum output power is $6 \mathrm{~mW}$, and the laser works up to $58 \mathrm{~K}$ in continuous wave. The emission is single mode at all injection currents and operating temperatures. The inherent linewidth of the QCL is less than $20 \mathrm{kHz}$ within a $4 \mathrm{~ms}$ integration time. It increases to $\sim 0.5 \mathrm{MHz}$ within an integration time of $300 \mathrm{~ms}$. This is sufficient for the Doppler limited gas phase spectroscopy of many molecules. For example, the Doppler limited linewidth 


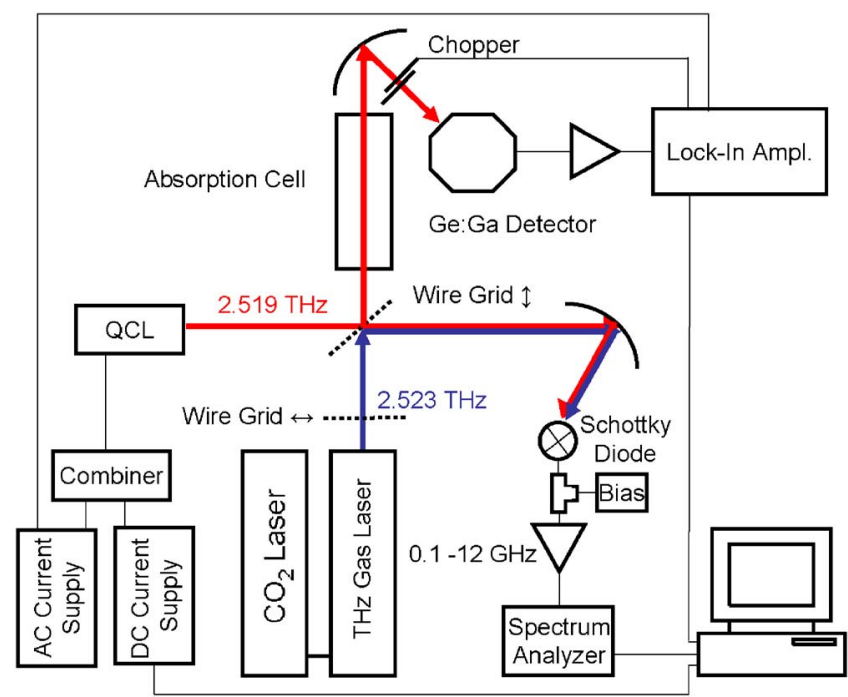

FIG. 1. (Color online) Terahertz spectrometer with QCL as radiation source.

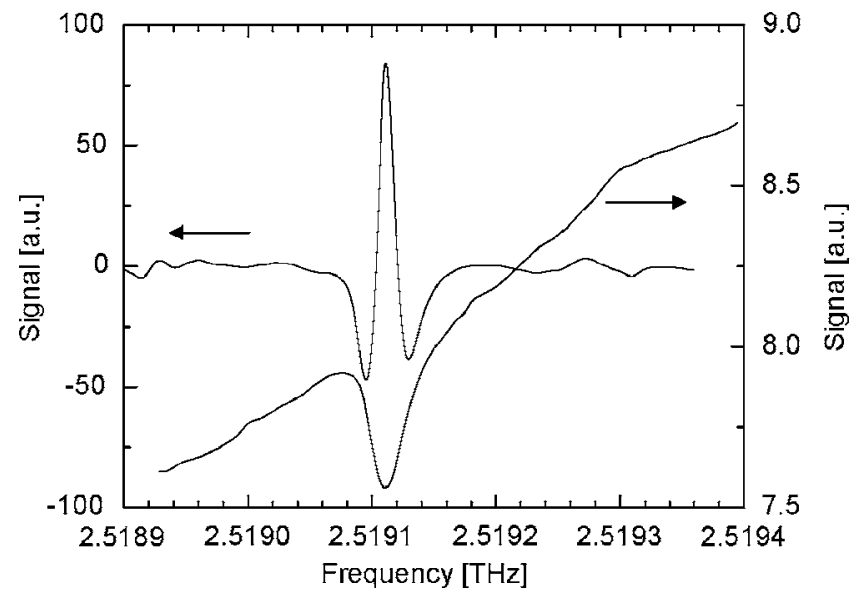

FIG. 2. Typical absorption spectra of the $\mathrm{CH}_{3} \mathrm{OH}$ transition $(p=150 \mathrm{~Pa}$ ) measured with amplitude modulation and frequency modulation. In the case of amplitude modulation the tilted base line is due to the change of power from the QCL with changing current. The base line drift can be almost completely removed by frequency modulation of the QCL.

point is measured. This is especially important because the frequency tuning of the QCL is not linear with current. A frequency range of $2.517-2.521 \mathrm{THz}$ is available from this QCL by changing temperature and current.

As a demonstration we have performed absorption spectroscopy of the $P$ transition $\left|E_{1}, v=0, n=2, K=2, J=20\right\rangle$ $\leftarrow\left|E_{1}, v=0, n=1, K=3, J=21\right\rangle$ of the methanol isotopomer ${ }^{12} \mathrm{CH}_{3}^{16} \mathrm{OH}$. Here $E_{1}$ is the symmetry species, $v$ is the vibrational state, $n$ denotes the torsional state, and $K$ and $J$ are the usual angular momentum quantum numbers. ${ }^{19}$ In Fig. 2 two typical spectra are shown. One is measured with amplitude modulation and the other is measured with frequency modulation. As can be seen the latter technique eliminates the tilted base line to a large extent. Figure 3 shows the absorption line along with a Voigt profile fitted to the measured profile. As can be seen from the residue (measurement minus fit) the agreement is very good. The self-pressure broadening of the methanol line was determined by measuring its profile at different pressures up to $1000 \mathrm{~Pa}$ and determining the full width at half maximum (FWHM). A least squares fit to the

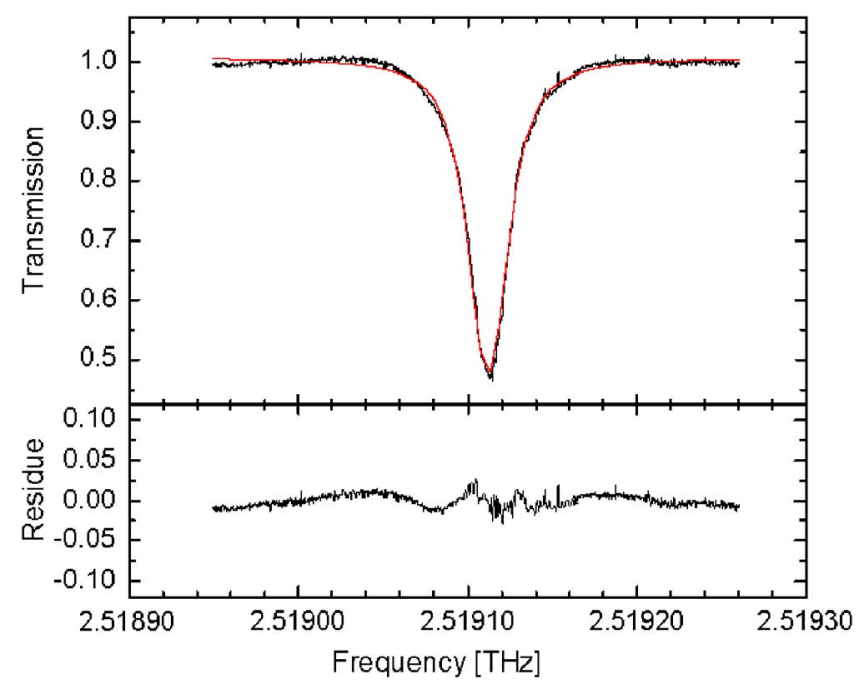

FIG. 3. (Color online) $\mathrm{CH}_{3} \mathrm{OH}$ absorption spectrum measured at $100 \mathrm{~Pa}$ after subtraction of the tilted base line. The straight line is a fit of a Voigt profile to the absorption line. The lower panel shows the residue after subtraction of the fit from the measured data. 


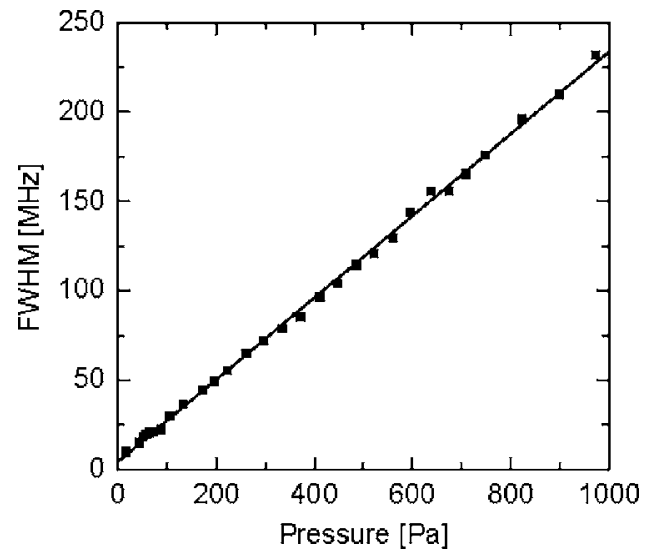

FIG. 4. FWHM of the $\mathrm{CH}_{3} \mathrm{OH}$ transition as a function of pressure.

FWHM data yields a pressure broadening coefficient of $229(2) \mathrm{kHz} / \mathrm{Pa}$ (Fig. 4). This is similar to pressure broadening coefficients of other methanol lines [265.6(2) kHz/Pa at $76 \mathrm{GHz}$ (Ref. 20) and $290 \mathrm{kHz} / \mathrm{Pa}$ at $2.524 \mathrm{THz}$ (Ref. 21)]. The center frequency of the absorption line was determined from least squares fits of a Voigt profile to the measured profile. In Fig. 5 the center frequency is plotted as a function of the pressure. The straight line is a least squares fit to the data. From this fit the pressure shift is determined to be $10(2) \mathrm{kHz} / \mathrm{Pa}$ and the zero pressure frequency is determined to be $2.519112(1) \mathrm{THz}$. Both values agree well with other published data $[6 \mathrm{kHz} / \mathrm{Pa}$ measured for a different line than the one here ${ }^{22}$ and 2.519 107(2) THz (Ref. 19)]. It is worth noting that the accuracy is not limited by the frequency stability or linewidth of the QCL because the typical error in the determination of the line peak from the fit is less than $\pm 150 \mathrm{kHz}$ (95\% rms). Instead, it is the frequency accuracy of the gas laser. This is limited by the precision with which it is possible to tune the gas laser line to its peak power (approximately $\pm 1 \mathrm{MHz}$ ).

In summary, we have realized a terahertz spectrometer for high-resolution gas phase spectroscopy based on a DFB QCL as the radiation source. Frequency calibration of the spectra is achieved by instantaneously measuring the frequency difference between the QCL and a terahertz gas laser.

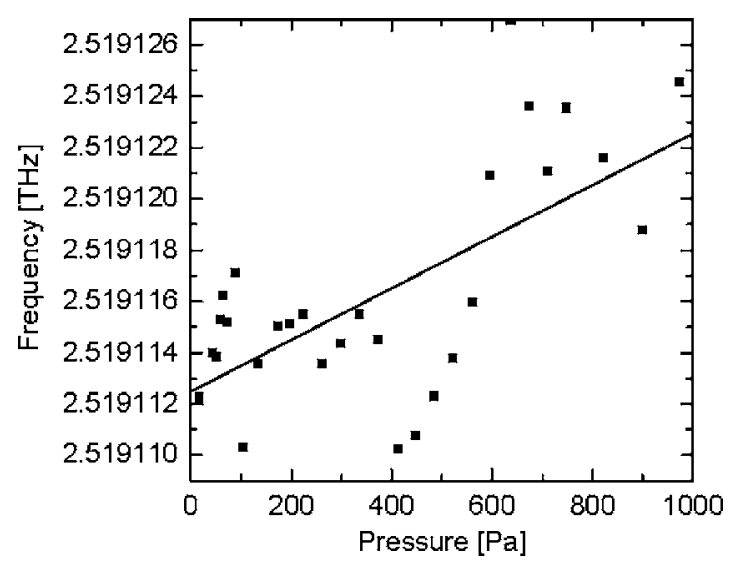

FIG. 5. Center frequency of the $\mathrm{CH}_{3} \mathrm{OH}$ transition as a function of pressure. Note that the scatter of the data is not caused by the QCL but is due to the inaccuracy of the gas laser frequency which provides the reference.
Amplitude as well as frequency modulation schemes have been realized. While the first technique yields the true line shape, the second allows removal of the tilted base line. By replacing the gas laser with a phase locked, frequency tunable, multiplied microwave source, the frequency measurement can be made more accurate as well as more broadband. We have measured the transition frequency, pressure broadening and pressure shift parameters of a methanol line at $2.5 \mathrm{THz}$. The comparison of our data with other spectroscopic data shows good agreement and demonstrates that the terahertz QCL is a very useful source for high-resolution gas phase spectroscopy.

This work was supported in part by the Investitionsbank Berlin and the European Commission through the PASR project "TeraSec," the IP project "Teranova," and the Marie Curie RTN "Poise."

${ }^{1}$ Spectroscopy from Space, edited by J. Demaison, K. Sarka, and E. H. Cohen (Kluwer, Dordrecht, 2001), Vol. 20.

${ }^{2}$ F. Lucia, in Sensing with Terahertz Radiation, edited by D. Mittleman (Springer, Berlin, 2003), Vol. 85, p. 39.

${ }^{3}$ K. M. Evenson, D. A. Jennings, and M. D. Vanek, in Frontiers of Laser Spectroscopy of Gases, edited by A. C. P. Alves, J. M. Brown, and J. M. Hollas (Kluwer, Dordrecht, 1988), Vol. 234, p. 43.

${ }^{4}$ P. Verhoeve, E. Zwart, M. Verslius, J. Ter Meulen, W. L. Meerts, A. Dymanus, and D. Mclay, Rev. Sci. Instrum. 61, 1612 (1990).

${ }^{5}$ G. A. Blake, K. B. Laughlin, R. C. Cohen, K. L. Busarow, D.-H. Gwo, C. A. Schmuttenmaer, D. W. Steyert, and R. J. Saykally, Rev. Sci. Instrum. 62, 1701 (1991).

${ }^{6}$ R. Gendriesch, F. Lewen, G. Winnewisser, and J. Hahn, J. Mol. Spectrosc. 203, 205 (2000).

${ }^{7}$ D. M. Mittleman, R. H. Jacobsen, R. Neelamani, R. G. Baraniuk, and M. C. Nuss, Appl. Phys. B: Lasers Opt. B67, 379 (1998).

${ }^{8}$ A. Bartels, A. Thoma, C. Janke, A. Dreyhaupt, S. Winnerl, and M. Helm, Opt. Express 14, 430 (2006).

${ }^{9}$ K. M. Evenson, R. J. Saykally, D. A. Jennings, R. F. Curl, Jr., and J. M. Brown, in Chemical and Biochemical Applications of Lasers, edited by C. Bradley Moore (Academic, New York, 1980), Vol. 5, p. 95.

${ }^{10}$ R. Köhler, A. Tredicucci, F. Beltram, H. E. Beere, E. H. Linfield, A. G. Davies, D. A. Ritchie, R. C. Iotti, and F. Rossi, Nature (London) 417, 156 (2002).

${ }^{11}$ A. Barkan, F. K. Tittel, D. M. Mittleman, R. Dengler, P. H. Siegel, G. Scalari, L. Ajili, J. Faist, H. E. Beere, E. H. Linfield, A. G. Davies, and D. A. Ritchie, Opt. Lett. 29, 575 (2004).

${ }^{12}$ S. Barbieri, J. Alton, H. E. Beere, E. H. Linfield, D. A. Ritchie, S. Withington, G. Scalari, L. Ajili, and J. Faist, Opt. Lett. 29, 1632 (2004).

${ }^{13}$ H.-W. Hübers, S. G. Pavlov, A. D. Semenov, R. Köhler, L. Mahler, A. Tredicucci, H. E. Beere, D. A. Ritchie, and E. H. Linfield, Opt. Express 13, 5890 (2005).

${ }^{14}$ B. S. Williams, S. Kumar, Q. Hu, and J. L. Reno, Electron. Lett. 42, 89 (2006).

${ }^{15}$ J. Faist, M. Beck, T. Aellen, and E. Gini, Appl. Phys. Lett. 78, 147 (2001).

${ }^{16}$ L. Mahler, A. Tredicucci, R. Köhler, F. Beltram, H. E. Beere, E. H. Linfield, and D. A. Ritchie, Appl. Phys. Lett. 87, 181101 (2005).

${ }^{17}$ N. G. Douglas, Millimetre and Submillimetre Wavelength Lasers (Springer, Berlin, 1989), Vol. 61, p. 73.

${ }^{18}$ H. P. Röser, H.-W. Hübers, T. W. Crowe, and W. C. B. Peatman, Infrared Phys. Technol. 35, 451 (1994).

${ }^{19}$ G. Moruzzi, F. Strumia, P. Carnesecche, B. Carli, and M. Parlotti, Infrared Phys. 29, 47 (1989).

${ }^{20}$ P. Minguzzi, M. Tonelli, G. Carrara, and A. Di Lieto, J. Mol. Spectrosc. 109, 395 (1985)

${ }^{21}$ A. D. Semenov, H.-W. Hübers, H. Richter, M. Birk, M. Krocka, U. Mair, K. Smirnov, G. Goltsman, and B. V. Voronov, Physica C 372-376, 448 (2002).

${ }^{22}$ G. Buffa, G. Moruzzi, and O. Tarrini, Infrared Phys. 27, 349 (1987). 